\title{
A New Modified Algorithm Based Upon Exponential and Polynomial Functions for Solving Initial Value Problems
}

\author{
Kamlesh Lohana Asif Ali Shaikh Sania Qureshi \\ Department of Basic Sciences and Related Studies, Mehran University of Engineering \& Technology, \\ Jamshoro, Sindh, Pakistan
}

\begin{abstract}
The main focus of this paper is to propose a fourth-order convergent modified numerical algorithm by using an exponential and polynomial type function for getting the approximate solution of initial value problems (IVPs) in ordinary differential equations (ODEs). The proposed algorithm has been tested and analyzed for the convergence, stability, consistency, of several numerical experiments that proved the superiority of the proposed algorithm against algorithms including the fourth-order arithmetic mean (AM), the fourth-order harmonic mean (HM), and the fourth-order Contra harmonic mean $\left(C_{o} M\right)$.
\end{abstract}

Keywords: Modified numerical algorithm, Ordinary differential equation, Order of convergence, Initial value problems, Stability, and Consistency.

DOI: $10.7176 / \mathrm{MTM} / 11-6-01$

Publication date: December $31^{\text {st }} 2021$

\section{Introduction}

Many researchers are working to solve many real-world physical problems. They are the ones that came up with the laws and theories that are related to natural physical problems. Most of the time, these laws take the form of differential equations. In the domains of engineering and physics, an ODE is crucial. It is the most significant tool for solving the physical and natural problems that belong to the field of science. The mostly real-world applications and pure mathematical research are interconnected to the ordinary differential equations. The most mathematical formulation of physical laws are defined in terms of ODEs and have applications in the field of fluid dynamics, chemistry, Biology, Medicine, Population dynamics, Economy, Social sciences, and many more. It is used to define the motion of projectile, rocket, charge, satellite planet, current in a circuit, conduction of heat, force on bodies, or bodies in motion depending on ODEs. There are many methods for solving initial value problems of the form given below:

$$
y^{\prime}=f(x, y), y\left(x_{0}\right)=y_{0}
$$

At present time, we have many exact and numerical techniques for solving the equation of the type (1) that gives approximate and analytical solutions, but some ODEs have no exact solutions. It is a known fact that several mathematical models arising from the real and physical life situations cannot be solved explicitly in most of the cases such as nonlinear lotka Volterra competition model and logistic equation in population dynamics, Lorenz system in meteorology, pendulum, and duffing equations in mechanical engineering, VanderPol equation in electrical engineering, Newton's law of cooling in thermodynamics, geodesic equation in geology, radioactive decay in nuclear physics, the motion of a charged particle, Fermi-Ulam-Pasta Oscillator and many more. In such situations, numerical methods of different characteristics and orders are needed mainly due to nonlinear terms involved in the practical problems.

These are all ODEs in which we need to use numerical methods to get the solutions. Some methods are explicit, and some are implicit methods. In continuation of this effort, many researchers have derived new numerical integration methods of explicit and implicit nature in an attempt to obtain better approximate results than various of the available ones in the present literature such as $[1,2,5,6,10,12]$, just to mention a few. In [10] author developed a new numerical technique for the solution of initial value problems of first-order ODEs whereas in the present study, we have proposed a new modified algorithm based upon the exponential and polynomial function for the solution of initial value problems of first-order ODEs, the paper is organized as follows: the Section 2 starts with the derivation of the polynomial and exponential type function for a proposed new modified numerical algorithm, the section 3 to proves that proposed new modified numerical algorithm is fourth-order convergent and discuss the local truncation error, consistency and larger stability region of the proposed algorithm structured within its subsections. The Section 4 takes into consideration four numerical problems of varying nature to illustrate the performance of the algorithm based on the computation of errors. The last Section 5 summarizes the above discussion with highlights of major findings in the present research study.

\subsection{Derivation of New modified algorithm}

Let us presume that the theoretical solution of $\mathrm{y}(\mathrm{x})$ to the initial value problem (1) can be locally represented in the interval $\left[x_{i}, x_{i+1}\right], i \geq 0$ by the exponential and polynomial type function;

$$
\varnothing(x)=\left(b_{1}+b_{2}\right) e^{-3 x}+b_{3} x^{3}+b_{4} x^{2}+b_{5} x+b_{6}
$$


where $b_{1}, b_{2}, b_{3}, b_{4}, b_{5}$ are real unknown coefficients and $b_{6}$ is a constant. We shall imagine $y_{n}$ is a numerical approximate to the theoretical outcome $\mathrm{y}(\mathrm{x})$ and. $\emptyset_{n}=\emptyset\left(x_{i}, y_{i}\right)$.

We interpret mesh point as follows:

$x_{i+1}=x_{i}+h, \quad i=0,1,2, \ldots$

Imposing the following constraints on the exponential and polynomial type function (2) in order to get the undetermined coefficients.

\subsection{The Exponential and Polynomial type Function.}

a. The exponential and polynomial function must correspond with the theoretical solution at

Hence we require that

$$
x=x_{i} \text { and } x=x_{i+1} \text {. }
$$

$\varnothing\left(x_{i}\right)=\left(b_{1}+b_{2}\right) e^{-3 x_{i}}+b_{3} x_{i}^{3}+b_{4} x_{i}^{2}+b_{5} x_{i}+b_{6}$.

$\emptyset\left(x_{i+1}\right)=\left(b_{1}+b_{2}\right) e^{-3 x_{i+1}}+b_{3} x_{i+1}^{3}+b_{4} x_{i+1}^{2}+b_{5} x_{i+1}+b_{6}$.

b. Secondly, the derivatives of the polynomial and exponential type function are required to correspond with the differential equation, furthermore its first, second, third and fourth derivatives with respect to $\mathrm{x}$ at $x=x_{i}$ we denote nth total derivatives of $\emptyset(x, y)$ with respect to $\mathrm{x}$ with $\emptyset^{(n)}$ such that

$\emptyset^{1}\left(x_{i}\right)=\theta_{n}$.

$\emptyset^{2}\left(x_{i}\right)=\theta_{n}^{(1)}$.

$\emptyset^{3}\left(x_{i}\right)=\theta_{n}^{(2)}$.

$\emptyset^{4}\left(x_{i}\right)=\theta_{n}^{(3)}$.

$\Theta_{n}=-3\left(b_{1}+b_{2}\right) e^{-3 x_{i}}+3 b_{3} x_{i}^{2}+2 b_{4} x_{i}+b_{5}$.

$\Theta_{n}^{(1)}=9\left(b_{1}+b_{2}\right) e^{-3 x_{i}}+6 b_{3} x_{i}+2 b_{4}$.

$\Theta_{n}^{(2)}=-27\left(b_{1}+b_{2}\right) e^{-3 x_{i}}+6 b_{3}$.

$\Theta_{n}^{(3)}=\left(b_{1}+b_{2}\right) e^{-3 x_{i}}$.

Solving $b_{1}+b_{2}$ from equation (13),

$\left(b_{1}+b_{2}\right)=\frac{1}{81} \Theta_{n}^{(3)} e^{3 x_{i}}$.

Substitute (14) into (12) we have,

$b_{3}=\frac{1}{6}\left(\theta_{n}^{(2)}+\frac{1}{3} \theta_{n}^{(3)}\right)$.

Substitute (14),(15) into (11), we have,

$b_{4}=\frac{1}{2}\left\{\Theta_{n}^{(1)}-\frac{1}{9} \Theta_{n}^{(3)}-\left(\Theta_{n}^{(2)}+\frac{1}{3} \Theta_{n}^{(3)}\right) x_{i}\right\}$.

Substitute (14),(15),(16) into (10) we have,

$b_{5}=\frac{1}{27} \theta_{n}^{(3)}-\left(\theta_{n}^{(1)}-\frac{1}{9} \theta_{n}^{(3)}\right) x_{i}+\frac{1}{2}\left(\theta_{n}^{(2)}+\frac{1}{3} \theta_{n}^{(3)}\right) x_{i}^{2}$.

Replace

$\emptyset\left(x_{i}\right)=y_{n}$.

$\emptyset\left(x_{i+1}\right)=y_{n+1}$,

implies that 
$\emptyset\left(x_{i+1}\right)-\emptyset\left(x_{i}\right)=y_{n+1}-y_{n}$.

$y_{n+1}-y_{n}=\left(b_{1}+b_{2}\right)\left[e^{-3 x_{i+1}}-e^{-3 x_{i}}\right]+b_{3}\left[x_{i+1}^{3}-x_{i}^{3}\right]+b_{4}\left[x_{i+1}^{2}-x_{i}^{2}\right]+b_{5}\left[x_{i+1}-x_{i}\right]$.

Recall that

$x_{i+1}=x_{i}+h$

with $\quad i=0,1,2 \ldots$

Substitute (14), (15), (16), (17) into (21), we have and neglecting the terms in $x_{i}, x_{i}^{2} \ldots$

$y_{n+1}=y_{n}+\frac{1}{81} \Theta_{n}^{(3)}\left(e^{-3 h}-1\right)+\frac{1}{6}\left(\Theta_{n}^{(2)}+\frac{1}{3} \theta_{n}^{(3)}\right) h^{3}+\frac{1}{2}\left(\theta_{n}^{(1)}-\frac{1}{9} \theta_{n}^{(3)}\right) h^{2}+\left(\theta_{n}+\frac{1}{27} \Theta_{n}^{(3)}\right) h$.

Hence (23) is the new modified algorithm for solution of the first order differential equation.

\subsection{Local Truncation Error Analysis}

We decide the order of convergence of any numerical algorithm by analysis of local truncation error. In order to check the order of the algorithm, we subtract the numerical algorithm (23) from the well-known Taylor's series expansion for $\mathrm{y}(\mathrm{x})$ in powers of $\mathrm{h}$ which has been described below:

We know that Taylor's series expansion is

$y\left(x_{n}\right)=y\left(x_{n}\right)+h \Theta+\frac{1}{2 !} h^{2} \Theta^{(1)}+\frac{1}{3 !} h^{3} \Theta^{(2)}+\frac{1}{4 !} h^{4} \Theta^{(3)}+\frac{1}{5 !} h^{5} \Theta^{(4)}+O\left(h^{6}\right)$.

Local truncation error is defined as

$L T E=Y\left(x_{n+1}\right)-y_{n+1}$.

$$
\begin{aligned}
\text { LTE }= & y\left(x_{n}\right)+h \Theta+\frac{1}{2 !} h^{2} \theta^{(1)}+\frac{1}{3 !} h^{3} \Theta^{(2)}+\frac{1}{4 !} h^{4} \Theta^{(3)}+\frac{1}{5 !} h^{5} \Theta^{(4)}-\left[y_{n}+\frac{1}{81} \Theta_{n}^{(3)}\left(e^{-3 h}-1\right)+\frac{1}{6}\left(\theta_{n}^{(2)}+\right.\right. \\
& \left.\left.\frac{1}{3} \Theta_{n}^{(3)}\right) h^{3}+\frac{1}{2}\left(\Theta_{n}^{(1)}-\frac{1}{9} \theta_{n}^{(3)}\right) h^{2}+\left(\Theta_{n}+\frac{1}{27} \theta_{n}^{(3)}\right) h\right]+O\left(h^{6}\right) .
\end{aligned}
$$

Replacing the terms $e^{-3 h}$ by its Maclaurin's series, one obtains.

$$
\begin{aligned}
& L T E=y\left(x_{n}\right)+h \Theta+\frac{1}{2 !} h^{2} \Theta^{(1)}+\frac{1}{3 !} h^{3} \theta^{(2)}+\frac{1}{4 !} h^{4} \Theta^{(3)}+\frac{1}{5 !} h^{5} \Theta^{(4)}-\left[y_{n}+\frac{1}{81} \Theta_{n}^{(3)}\left(1-3 h+\frac{(-3 h)^{2}}{2 !}+\frac{(-3 h)^{3}}{3 !}+\right.\right. \\
& \left.\left.\frac{(-3 h)^{4}}{4 !}+\frac{(-3 h)^{5}}{5 !}+\cdots-1\right)+\frac{1}{6}\left(\Theta_{n}^{(2)}+\frac{1}{3} \Theta_{n}^{(3)}\right) h^{3}+\frac{1}{2}\left(\Theta_{n}^{(1)}-\frac{1}{9} \Theta_{n}^{(3)}\right) h^{2}+\left(\Theta_{n}+\frac{1}{27} \theta_{n}^{(3)}\right) h\right]+O\left(h^{6}\right) \text {. } \\
& L T E=y\left(x_{n}\right)+h \theta+\frac{1}{2 !} h^{2} \theta^{(1)}+\frac{1}{3 !} h^{3} \theta^{(2)}+\frac{1}{4 !} h^{4} \theta^{(3)}+\frac{1}{5 !} h^{5} \theta^{(4)}-\quad\left[y_{n}+\frac{1}{81} \Theta_{n}^{(3)}\left(-3 h+\frac{9 h^{2}}{2}+\frac{27 h^{3}}{6}+\right.\right. \\
& \left.\left.\frac{81 h^{4}}{24}+\frac{243 h^{5}}{120}\right)+\frac{1}{6}\left(\Theta_{n}^{(2)}+\frac{1}{3} \Theta_{n}^{(3)}\right) h^{3}+\frac{1}{2}\left(\Theta_{n}^{(1)}-\frac{1}{9} \Theta_{n}^{(3)}\right) h^{2}+\left(\Theta_{n}+\frac{1}{27} \Theta_{n}^{(3)}\right) h\right]+O\left(h^{6}\right) \text {. }
\end{aligned}
$$

Under local assumption the terms up to $h^{4}$ have been cancelled from (28) and we are left with the following expression:

$L T E=\frac{1}{5 !} h^{5}\left(\Theta_{n}^{(4)}-3 \Theta_{n}^{(3)}\right)+O\left(h^{6}\right)$.

Thus, the leading term of the local truncation error involves $h^{5}$ which confirms that the proposed new numerical modified algorithm based upon polynomial and exponential function given by (29) has fourth order of convergence.

\subsection{Consistency Analysis}

The numerical algorithm needs to be consistent that the truncation errors to be zero when the step size $h$ gets smaller and ultimately extend to zero. Among many, the consistency analysis of a numerical algorithm is one of the ways to check that whether

$\lim _{h \rightarrow 0} \frac{L T E}{h}=0$.

From the above criterion, it is easy to observe that the proposed modified numerical algorithm has consistency characteristics. Further, it is also known that any algorithm considered to be consistent has an order of accuracy greater than 0 .

Based upon this fact, consistency of the proposed algorithm can safely be claimed since the fourth-order accuracy of the proposed algorithm has already been proved in the previous section.

\subsection{Stability}

The algorithm is said to be numerically stable if it is efficient of damping out the small change execute in the input data. The idea of stability may be taken in different conditions: it may be correlated with the specific numerical algorithm used, or the step size $h$ used in numerical computations or with the particular problem being solved, one 
of the popular way to apply the test problem for stability analysis of the proposed numerical algorithm is $\left(\frac{d y}{d x}\right) y(x)=\emptyset y(x) ; y(0)=1$ which has known as exact solution $y(x)=\exp (-\emptyset x)$ where $\emptyset>0$ is, in general, a complex constant. For an integration interval $\left[x_{i}, x_{i+1}\right]$, where $h=x_{i}-x_{i+1}$; the exact solution at the point $x=$ $x_{i+1}$ is:

$y\left(x_{i+1}\right)=e^{\emptyset x_{i+1}}=e^{\emptyset\left(x_{i}+h\right)}=e^{\emptyset x_{i}} e^{\emptyset h}=y\left(x_{i}\right) e^{\emptyset h}, \emptyset<0$

When applied the proposed numerical algorithm on this test problem; it yields

$\boldsymbol{y}_{\boldsymbol{i}+\mathbf{1}}=\boldsymbol{\psi} \boldsymbol{y}_{\boldsymbol{i}}$ where $\boldsymbol{\psi}=\mathbf{1}+\emptyset \boldsymbol{h}+\frac{(\varnothing \boldsymbol{h})^{2}}{2}+\frac{(\varnothing \boldsymbol{h})^{3}}{\mathbf{6}}+\frac{(\varnothing \boldsymbol{h})^{\mathbf{4}}}{\mathbf{2 4}}$

Comparing the Equations (31) and (32), it is clearly seen that the Equation (32) is a five term approximation for the function $e^{\emptyset h}$ in the exact solution. The error growth factor given by (32) can be controlled by $|\psi|<1$ so that the errors may not amplify. Thus, the stability function of the proposed numerical algorithm (23) requires that

Setting $z=\varnothing h$, then (32) yields $\left|\mathbf{1}+\mathbf{z}+\frac{z^{2}}{\mathbf{2}}+\frac{z^{3}}{\mathbf{6}}+\frac{z^{4}}{\mathbf{2 4}}\right|<\mathbf{1}$

$$
\left|1+\emptyset h+\frac{(\varnothing h)^{2}}{2}+\frac{(\varnothing h)^{3}}{6}+\frac{(\varnothing h)^{4}}{24}\right|<1
$$

The region of absolute stability for the proposed numerical algorithm (23) is defined by the region in the complex plane such that $\left|\mathbf{1}+z+\frac{z^{2}}{2}+\frac{z^{3}}{6}+\frac{z^{4}}{24}\right|<1$.

The stability region is plotted in the figure 1 given below.

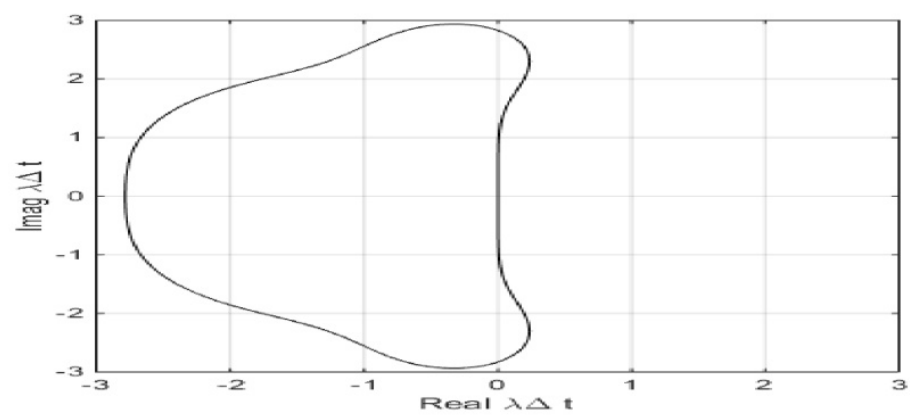

Figure 1. The Stability Region of the Fourth-Order Proposed Numerical Algorithm

\section{NUMERICAL PROBLEMS}

Examples 1-4 in this part discuss numerical values of $y(x)$ for initial value problems (IVPs) and absolute inaccuracy of the proposed algorithm and a few known algorithms. The results of the proposed new modified algorithm based on exponential and polynomial functions, AM (The fourth-order arithmetic mean), (The fourth-order harmonic mean), (The fourth-order Contra harmonic mean method), and their absolute percentage errors and exact solution at various $\mathrm{x}$-values are shown in Tables 1-4. These findings were validated using the MATLAB software (version 5.0). (R-2014-b).

Problem 4.1 consider initial value problem

$$
\frac{d y}{d x}=x y^{3}-y, y(0)=1, \quad 0 \leq x \leq 1, \quad h=0.1
$$

The exact solution of problem 4.1 is

$$
y(x)=\frac{2}{\sqrt{2+4 x+2 e^{2 x}}}
$$


Table 1. Shows the results of example 4.1

\begin{tabular}{|l|l|l|l|l|l|l|l|l|l|}
\hline X-Value & Exact & Proposed & Error & AM & Error & HM & Error & $\mathbf{C}_{\mathbf{0}} \mathbf{M}$ & Error \\
\hline 0 & 1.0000 & 1.0000 & 0 & 1.0000 & 0.0002 & 1.0000 & 0 & 1.0000 & 0 \\
\hline 0.1000 & 0.9088 & 0.9088 & 0.000 & 0.9090 & 0.0002 & 0.9087 & 0.0001 & 0.9088 & 0.0001 \\
\hline 0.2000 & 0.8316 & 0.8316 & 0.000 & 0.8319 & 0.0003 & 0.8314 & 0.0002 & 0.8315 & 0.0001 \\
\hline 0.3000 & 0.7645 & 0.7645 & 0.000 & 0.7648 & 0.0003 & 0.7643 & 0.0002 & 0.7643 & 0.0001 \\
\hline 0.4000 & 0.7049 & 0.7049 & 0.000 & 0.7052 & 0.0003 & 0.7046 & 0.0002 & 0.7047 & 0.0001 \\
\hline 0.5000 & 0.6511 & 0.6511 & 0.000 & 0.6514 & 0.0003 & 0.6508 & 0.0003 & 0.6509 & 0.0002 \\
\hline 0.6000 & 0.6019 & 0.6019 & 0.000 & 0.6023 & 0.0003 & 0.6017 & 0.0003 & 0.6018 & 0.0002 \\
\hline 0.7000 & 0.5566 & 0.5566 & 0.000 & 0.5570 & 0.0003 & 0.5564 & 0.0003 & 0.5565 & 0.0002 \\
\hline 0.8000 & 0.5146 & 0.5146 & 0.000 & 0.5149 & 0.0003 & 0.5143 & 0.0003 & 0.5144 & 0.0002 \\
\hline 0.9000 & 0.4754 & 0.4754 & 0.000 & 0.4757 & 0.0003 & 0.4751 & 0.0003 & 0.4752 & 0.0002 \\
\hline 1.0000 & 0.4388 & 0.4388 & 0.000 & 0.4391 & 0.0003 & 0.4385 & 0.0002 & 0.4386 & 0.0002 \\
\hline
\end{tabular}

Problem 4.2 consider initial value problem

$$
\frac{d y}{d x}=x+y, y(0)=1, \quad 0 \leq x \leq 1, \quad h=0.1
$$

The exact solution of problem 4.2 is

$$
y(x)=2 e^{x}-x-1
$$

Table 2. Shows the results of example 4.2

\begin{tabular}{|l|l|l|l|l|l|l|l|l|l|}
\hline X-Value & Exact & Proposed & Error & AM & Error & HM & Error & $\mathbf{C}_{\mathbf{0}} \mathbf{M}$ & Error \\
\hline 0 & 1.0000 & 1.0000 & 0 & 1.0000 & 0 & 1.000 & 0 & 1.0000 & 0 \\
\hline 0.1000 & 1.1103 & 1.1103 & 0.0000 & 1.1100 & 0.0003 & 1.1105 & 0.0001 & 1.1104 & 0.0001 \\
\hline 0.2000 & 1.2428 & 1.2428 & 0.0000 & 1.2421 & 0.0007 & 1.2431 & 0.0003 & 1.2430 & 0.0002 \\
\hline 0.3000 & 1.3997 & 1.3997 & 0.0000 & 1.3986 & 0.0011 & 1.4001 & 0.0004 & 1.4000 & 0.0002 \\
\hline 0.4000 & 1.5836 & 1.5836 & 0.0000 & 1.5821 & 0.0015 & 1.5842 & 0.0006 & 1.5840 & 0.0003 \\
\hline 0.5000 & 1.7974 & 1.7974 & 0.0000 & 1.7955 & 0.0020 & 1.7982 & 0.0008 & 1.7979 & 0.0004 \\
\hline 0.6000 & 2.0442 & 2.0442 & 0.0000 & 2.0417 & 0.0025 & 2.0452 & 0.0009 & 2.0447 & 0.0005 \\
\hline 0.7000 & 2.3275 & 2.3275 & 0.0000 & 2.3244 & 0.0031 & 2.3286 & 0.0011 & 2.3281 & 0.0006 \\
\hline 0.8000 & 2.6511 & 2.6511 & 0.0000 & 2.6473 & 0.0038 & 2.6524 & 0.0014 & 2.6518 & 0.0007 \\
\hline 0.9000 & 3.0192 & 3.0192 & 0.0000 & 3.0146 & 0.0046 & 3.0208 & 0.0016 & 3.0201 & 0.0009 \\
\hline 1.0000 & 3.4366 & 3.4365 & 0.0000 & 3.4311 & 0.0054 & 3.4384 & 0.0019 & 3.4376 & 0.0010 \\
\hline
\end{tabular}

Problem 4.3 consider initial value problem

$$
\frac{d y}{d x}=x y^{2}-y, y(0)=1, \quad 0 \leq x \leq 1, \quad h=0.1
$$

The exact solution of problem 4.3 is

$$
y(x)=\frac{1}{x+1}
$$

Table 3. Shows the results of example 4.3

\begin{tabular}{|l|l|l|l|l|l|l|l|l|l|}
\hline X-Value & Exact & Proposed & Error & AM & Error & HM & Error & $\mathbf{C}_{\mathbf{0}} \mathbf{M}$ & Error \\
\hline 0 & 1.0000 & 1.0000 & 0 & 1.0000 & 0 & 1.0000 & 0 & 1.0000 & 0.0001 \\
\hline 0.1000 & 0.9091 & 0.9091 & 0.0000 & 0.9093 & 0.0002 & 0.9090 & 0.0001 & 0.9090 & 0.0001 \\
\hline 0.2000 & 0.8333 & 0.8333 & 0.0000 & 0.8337 & 0.0004 & 0.8331 & 0.0002 & 0.8332 & 0.0002 \\
\hline 0.3000 & 0.7692 & 0.7692 & 0.0000 & 0.7697 & 0.0004 & 0.7689 & 0.0003 & 0.7691 & 0.0002 \\
\hline 0.4000 & 0.7143 & 0.7143 & 0.0000 & 0.7148 & 0.0005 & 0.7139 & 0.0003 & 0.7141 & 0.0002 \\
\hline 0.5000 & 0.6667 & 0.6667 & 0.0000 & 0.6672 & 0.0006 & 0.6663 & 0.0004 & 0.6665 & 0.0002 \\
\hline 0.6000 & 0.6250 & 0.6250 & 0.0000 & 0.6256 & 0.0006 & 0.6246 & 0.0004 & 0.6248 & 0.0002 \\
\hline 0.7000 & 0.5882 & 0.5882 & 0.0000 & 0.5889 & 0.0006 & 0.5878 & 0.0004 & 0.5880 & 0.0002 \\
\hline 0.8000 & 0.5556 & 0.5556 & 0.0000 & 0.5562 & 0.0007 & 0.5551 & 0.0004 & 0.5553 & 0.0003 \\
\hline 0.9000 & 0.5263 & 0.5263 & 0.0000 & 0.5270 & 0.0007 & 0.5258 & 0.0005 & 0.5260 & 0.0003 \\
\hline 1.0000 & 0.5000 & 0.5000 & 0.0000 & 0.5007 & 0.0007 & 0.4995 & 0.0005 & 0.4997 & 0.0004 \\
\hline
\end{tabular}

Problem 4.4 consider initial value problem

$$
\frac{d y}{d x}=\frac{y^{3} \cos x}{2}, y(0)=1, \quad 0 \leq x \leq 1, \quad h=0.1
$$

The exact solution of problem 4.4 is

$$
y(x)=\frac{1}{\sqrt{1-\sin x}}
$$


Table 4. Shows the results of example 4.4

\begin{tabular}{|l|l|l|l|l|l|l|l|l|l|}
\hline X-Value & Exact & Composed & Error & AM & Error & HM & Error & $\mathbf{C}_{\mathbf{0}} \mathbf{M}$ & Error \\
\hline 0 & 1.0000 & 1.0000 & 0 & 1.0000 & 0 & 1.0000 & 0 & 1.0000 & 0 \\
\hline 0.1000 & 1.0540 & 1.0540 & 0.0000 & 1.0539 & 0.0001 & 1.0540 & 0.0000 & 1.0540 & 0.0000 \\
\hline 0.2000 & 1.1171 & 1.1172 & 0.0001 & 1.1170 & 0.0001 & 1.1171 & 0.0000 & 1.1171 & 0.0000 \\
\hline 0.3000 & 1.1914 & 1.1915 & 0.0001 & 1.1912 & 0.0002 & 1.1914 & 0.0000 & 1.1914 & 0.0000 \\
\hline 0.4000 & 1.2798 & 1.2799 & 0.0002 & 1.2794 & 0.0003 & 1.2797 & 0.0000 & 1.2797 & 0.0000 \\
\hline 0.5000 & 1.3860 & 1.3862 & 0.0002 & 1.3855 & 0.0005 & 1.3859 & 0.0001 & 1.3859 & 0.0001 \\
\hline 0.6000 & 1.5156 & 1.5159 & 0.0003 & 1.5148 & 0.008 & 1.5154 & 0.0002 & 1.5154 & 0.0002 \\
\hline 0.7000 & 1.6765 & 1.6770 & 0.0005 & 1.6753 & 0.0012 & 1.6762 & 0.0003 & 1.6762 & 0.0003 \\
\hline 0.8000 & 1.8810 & 1.8816 & 0.0006 & 1.8790 & 0.0020 & 1.8804 & 0.0005 & 1.8803 & 0.0006 \\
\hline 0.9000 & 2.1483 & 2.1490 & 0.0007 & 2.1449 & 0.0034 & 2.1475 & 0.0008 & 2.1471 & 0.00012 \\
\hline 1.0000 & 2.5116 & 2.5119 & 0.0003 & 2.5054 & 0.0062 & 2.5105 & 0.0011 & 2.5091 & 0.00025 \\
\hline
\end{tabular}

We notice that in Tables 1-4, the error incurred in the fourth-order arithmetic mean (AM), The fourth-order harmonic mean $(\mathrm{HM})$ and The fourth-order Contra harmonic mean $\left(\mathrm{C}_{0} \mathrm{M}\right)$ is greater than that of the proposed numerical algorithm and the same time get larger as $\boldsymbol{n}$ increases. Hence proposed numerical algorithm is more accurate than the fourth-order arithmetic mean (AM), The fourth-order harmonic mean (HM), and The fourthorder Contra harmonic mean $\left(\mathrm{C}_{0} \mathrm{M}\right)$ as we can see from Tables 1-4.

\section{CONCLUSION}

In this research work, a new modified numerical algorithm has been proposed for solving the IVPs in ODEs (ordinary differential equations). The proposed algorithm has been created from exponential and polynomial type functions. The outcomes of the proposed algorithm have been compared with the fourth-order arithmetic means (AM), the fourth-order harmonic mean ( $\mathrm{HM})$, and the fourth-order Contra harmonic mean $\left(\mathrm{C}_{0} \mathrm{M}\right)$, and found that the proposed algorithm has been fourth-order and performing greater accuracy as compared to all mentioned algorithms and others well known second-order algorithms and third-order algorithms. The proposed numerical algorithm has also been analyzed for stability, consistency, and the local truncation error; and found that the proposed algorithm is more stable, consistent, and fourth-order accurate.

\section{REFERENCES}

1. Fadugba, S. E., Ogunyebi, S. N., \&Falodun, B. O. (2020). An Examination of a Second Order Numerical Method for Solving Initial Value Problems. Journal of the Nigerian Society of Physical Sciences, 120-127.

2. Oboyi, J., Ekoro, S. E., \&Bukie, P. T. (2019). Numerical solution of initial value problems by rational interpolation method using Chebyshev polynomials. Global Journal of Pure and Applied Sciences, 25(2), 185-194.

3. Fadugba, S. E., \& Idowu, J. O. (2019). Analysis of the properties of a third order convergence numerical method derived via transcendental function of exponential form. International Journal of Applied Mathematics and Theoretical Physics, 5(4), 97

4. Fadugba, S. E. (2019). Numerical technique via interpolating function for solving second order ordinary differential equations. Journal of Mathematics and Statistics, 1(2), 1-6.

5. Emmanuel, F. S., \& Qureshi, S. (2019). Convergent numerical method using transcendental function of exponential type to solve continuous dynamical systems. Journal of Mathematics (ISSN 1016-2526), 51(10), 45-56.

6. Qureshi, S., \& Emmanuel, F. S. (2018). Convergence of a numerical technique via interpolating function to approximate physical dynamical systems. Journal of Advanced Physics, 7(3), 446-450.

7. Emmanuel, F. S., \& Olumide, F. B. (2017). Development of a new one-step scheme for the solution of initial value problem (IVP) in ordinary differential equations. International Journal of Theoretical and Applied Mathematics, 3(2), 58.

8. Fadugba, S. E., \& Ajayi, A. O. (2017). Comparative study of a new scheme and some existing methods for the solution of initial value problems in ordinary differential equations. International Journal of Engineering and Future Technology, 14(3), 47-56.

9. Fadugba, S. E., \&Okunlola, J. T. (2017). Performance measure of a new one-step numerical technique via interpolating function for the solution of initial value problem of first order differential equation. World Scientific News, 90, 77-87.

10. SO, A., \&Ibijola, E. A. (2015). A new numerical method for solving first order differential equations. American journal of applied mathematics and statistics, 3(4), 156-160.

11. Abolarin, O. E., \&Akingbade, S. W. Nigerian Journal of Mathematics and Applications Volume24,(2015), 261-267 cGNig. J. Math. Appl. http://www. njmaman. com A NEW NUMERICAL METHOD FOR THE 


\section{SOLUTION OF INITIAL VALUE PROBLEMS}

12. Ochoche, A. (2007). Improving the modified Euler method. Leonardo Journal of Sciences, 10, 1-8.

13. Kama, P., \&Ibijola, E. A. (2001). On a new one-step method for numerical solution of initial-value problems in ordinary differential equations. International journal of computer mathematics, 77(3), 457-467.

14. bin Yaakub, A. R. (1996). Computer solution of non-linear integration formula for solving initial value problems (Doctoral dissertation, Loughborough University). 